Orthopäde $2021 \cdot 50: 587-588$

https://doi.org/10.1007/s00132-021-04123-7

Online publiziert: 22. Juni 2021

๑) Der/die Autor(en) 2021

Markus Rupp ${ }^{1}$ Maximilian Kerschbaum ${ }^{1}$ Viola Freigang ${ }^{1}$ Susanne Bärtl ${ }^{1}$. Florian Baumann ${ }^{1} \cdot$ Andrej Trampuz $^{2} \cdot$ Volker Alt $^{1}$

' Klinik und Poliklinik für Unfallchirurgie, Universitätsklinikum Regensburg (UKR), Regensburg, Deutschland

${ }^{2}$ Charité - Universitätsmedizin Berlin und Center for Musculoskeletal Surgery (CMSC), Berlin, Deutschland

\title{
Erratum zu: PJI-TNM als neues Klassifikationssystem für Endoprotheseninfektionen
}

\section{Eine Evaluation von 20 Fällen}

\section{Erratum zu:}

Orthopäde 2020

https://doi.org/10.1007/s00132-020-

03933-5

Der Artikel „PJI-TNM als neues Klassifikationssystem für Endoprotheseninfektionen. Eine Evaluation von 20 Fällen“ von Markus Rupp, Maximilian Kerschbaum, Viola Freigang, Susanne Bärtl, Florian Baumann, Andrej Trampuz und Volker Alt wurde ursprünglich Online First ohne „Open Access“ auf der Internetplattform des Verlags publiziert. Nach der Veröffentlichung in Bd. 50 Heft 3 pp. 198-206 hatten sich die Autoren für eine "Open Access“Veröffentlichung entschieden. Das Urheberrecht des Artikels wurde deshalb in (c) The Author(s) 2020 geändert.

\section{Korrespondenzadresse}

Prof. Dr. med. Dr. biol. hom. Volker Alt

Klinik und Poliklinik für Unfallchirurgie, Universitätsklinikum Regensburg (UKR) Franz-Josef-Strauß-Allee 11, 93053 Regensburg, Deutschland volker.alt@ukr.de

Förderung. Open Access funding enabled and organized by Projekt DEAL.

Open Access. Dieser Artikel wird unter der Creative Commons Namensnennung 4.0 International Lizenz veröffentlicht, welche die Nutzung, Vervielfältigung, Bearbeitung, Verbreitung und Wiedergabe in jeglichem Medium und Format erlaubt, sofern Sie den/die ursprünglichen Autor(en) und die Quelle ordnungsgemäß nennen, einen Link zur Creative Commons Lizenz beifügen und angeben, ob Änderungen vorgenommen wurden.
Die in diesem Artikel enthaltenen Bilder und sonstiges Drittmaterial unterliegen ebenfalls der genannten Creative Commons Lizenz, sofern sich aus der Abbildungslegende nichts anderes ergibt. Sofern das betreffende Material nicht unter der genannten Creative Commons Lizenz steht und die betreffende Handlung nicht nach gesetzlichen Vorschriften erlaubt ist, ist für die oben aufgeführten Weiterverwendungen des Materials die Einwilligung des jeweiligen Rechteinhabers einzuholen.

Weitere Details zur Lizenz entnehmen Sie bitte der Lizenzinformation auf http://creativecommons.org/ licenses/by/4.0/deed.de.
Die Online-Version des Originalartikels ist unter https://doi.org/10.1007/s00132-020-03933-5 zu finden. 


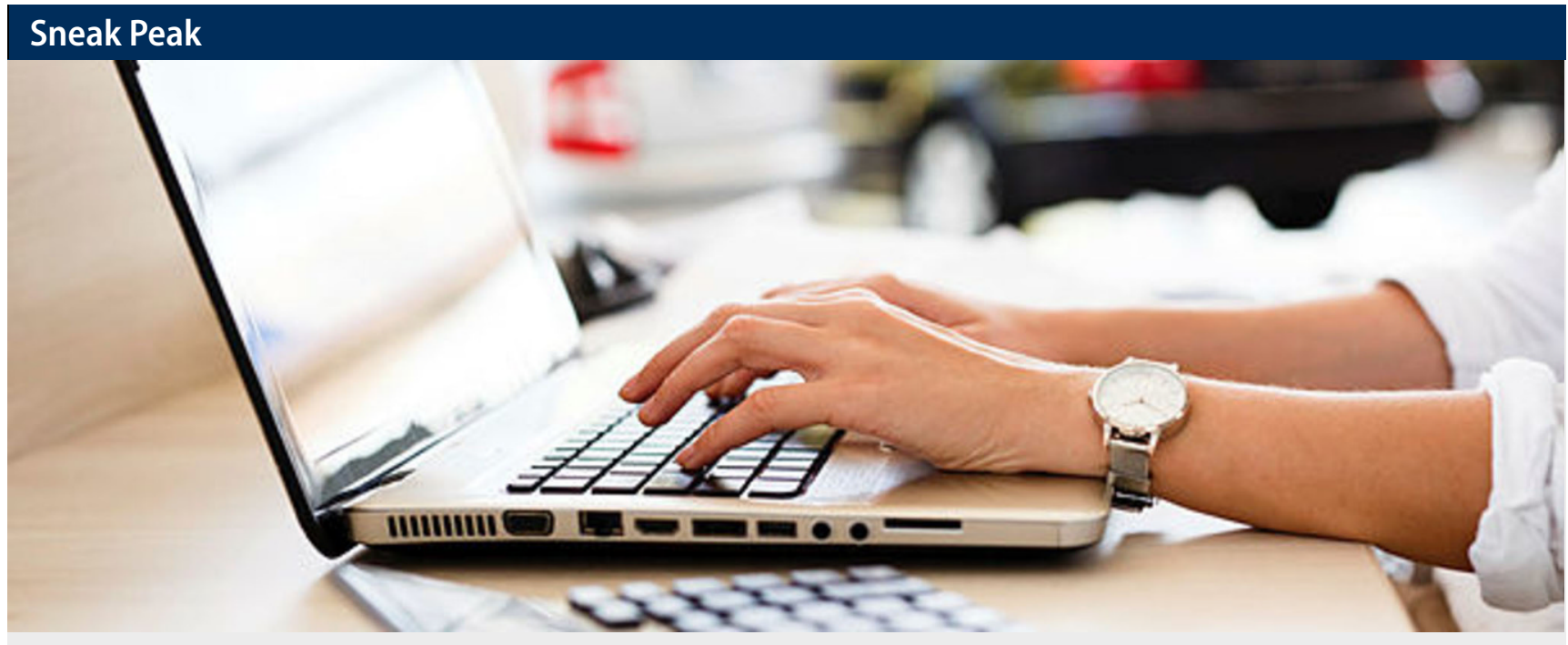

\section{Immer auf dem neuesten Stand - OnlineFirst-Publikationen in Der Orthopäde}

\section{Mit der OnlineFirst-Publikation von Springer Medizin sind die neuesten Artikel aus DerOrthopäde bereits vor Druck online im Volltext für Sie verfügbar - auch als PDF zum Download.}

Lesen Sie unter anderem:

\section{Originalien}

- Manuelle Beimischung von Antibiotika zu industriellen Knochenzementmischungen (Mustafa Citak, Stefan Luck, Philip Linke, Thorsten Gehrke, Klaus-Dieter Kühn)

- Kostendeckung bei komplexen pädiatrischen Wirbelsäulendeformitäten mit präoperativer Halotraktion - ein wirtschaftliches Problem? (N. von Dercks, A. Völker, E. Schumann, N. H. von der Höh, C. E. Heyde )

- Patientensicherheit in der endoprothetischen Weiterbildung (S. Rohe, S. Brodt, C. Windisch, G. Matziolis, S. Böhle )

- Vergleich der DRG-Erlöse zwischen Fast- und Slow-Track-Verfahren beim zweizeitigen Prothesenwechsel bei periprothetischen Hüftinfektionen im aG-DRG-System 2020 (Katja Hierl, Markus Rupp, Michael Worlicek, Florian Baumann, Christian Pfeifer, Volker Alt)

- Referenzwerte in der isometrischen Kraftdiagnostik (Aki Pietsch, Jan Schröder, Rüdiger Reer, Christopher Edler, Andrej Kutasow, Helge Riepenhof)

- Protheseninfektionen und die zunehmende Bedeutung psychologischer Komorbiditäten (Nike Walter, Markus Rupp, Thilo Hinterberger, Volker Alt)

- Chirurgische Aufklärung: Klar geregelt durch das Patientenrechtegesetz - deutliche Unsicherheit bei Medizinstudierenden (R. J. Seemann, P. Melcher, C. Eder, J. Deckena, R. Kasch, S. Fröhlich, M. März, AG Lehre DGOU, M. Ghanem)

\section{Übersichten}

- Erschwerte Diagnosestellung eines Osteoidosteoms der Großzehe (Ruth Thiemann, Hans-Werner Seide, Klaus-Dieter Luitjens, Frank Timo Beil, Tim Rolvien, Lara Krüger )

- Muskuloskelettale Labordiagnostik im Leistungssport (Maximilian M. Delsmann, Julian Stürznickel, Michael Amling, Peter Ueblacker, Tim Rolvien)

\section{Videobeitrag: Aktuelle Operationstechniken}

- Polymethylmethacrylat-Zementbeschichtung intramedullärer Implantate (Markus Rupp, Nike Walter, Abdullah Ismat, Volker Alt)

Unter dem Zusatz "epub ahead of print" können OnlineFirst-Artikel bereits zitiert werden.

Alle Beiträge finden Sie im Online-Archiv auf: https://www.springermedizin.de/derorthopaede 